\title{
Le problème de la normalisation d'une rivière en connexion avec les mouvements des eaux dans le domaine du Delta
}

\author{
The problem of restoring normal conditions \\ in a river affected by movements of water \\ in the Delta area
}

\author{
PAR H. M. OUDSHOORN,
}

INGENIEUR DE LA SECTION D'ETUDES HYDRAULIOUES FLUVIALES, SERVICES DU DELTA, RIJLSWATERSTAAT, LA HAYE

\begin{abstract}
Lorsqu'en 1968 les écluses d'évacuation du Haringvliet seront mises en service, le régime des courants d'eall dans la division septentrionale du bassin de l'estuaire du Delta subira des changements radicaux, ce qui entrainera certaines conséquences pour la stabilité du fond ainsi que pour la navigation.

La bifurcation des fleuves au droit de Dordrecht présente un exemple particulièrement intéressant grâce d̀ la circonstance que ce carrefour fluvial fait partie de l'itinéraire très fréquenté de la batellerie qui se dirige de Rotterdam vers le haut Rhin. En effet, dans les branches fréquentées, des variations de phases des courants sont à prévoir à tel point que la navigation sera gravement incommodée à moins que des travaux de réadaplation soient réalisés.

Les études, qui ont été consacrées à l'améliora. tion de celte bifurcation et à la nouvelle situation à réaliser, sont présentées dans cette communication.
\end{abstract}

Lorsque la fermeture des grands estuaires au sud-ouest des Pays-Bas sera achevée, les grands raz-de-marée dans cette région appartiendront au passé et le but essentiel du Projet Delta sera réalisé.

M. Bischoff van Heemskerck exposera plus longuement les problèmes qui jouent un rôle dans la fermeture même des bras de mer. J'aimerais toutefois parler avec plus de détails des changements que les barrages entraîneront dans

\begin{abstract}
The flow pattern in the northern division of the Delta estuary basin may be expected to undergo radical changes in 1968, when the discharge locks on the Haringuliet river go into service, which will affect both the bed stability and navigation conditions in the river.

The river fork at Dordrecht is a particularly interesting point in this respect, as it lies on the very busy shipping route leading from Rotterdam to the Upper Rhine. Unless suitable corrective measures are taken, current phase variations in the busier stretches of the river are likely to be so pronounced as to seriously affect shipping.
\end{abstract}

This article describes the research done on the problem, of improving the river fork and restoring it to satisfactory conditions.

le régime fluvial du Delta et expliquer cette nouvelle situation en prenant, comme exemple frappant de l'adaptation d'un fleuve, le point de séparation d'un certain nombre d'affluents du Rhin inférieur.

Comme M. de Rouville vient de le dire, le contact entre les eaux du Delta et la mer ne pourra pas être supprimé entièrement. En vue d'évacuer le débit du Rhin et de la Meuse, le barrage $d u$ « Haringvliet » comportera un 


\section{HAUTEUR DES MARÉES TEMPÉTE}

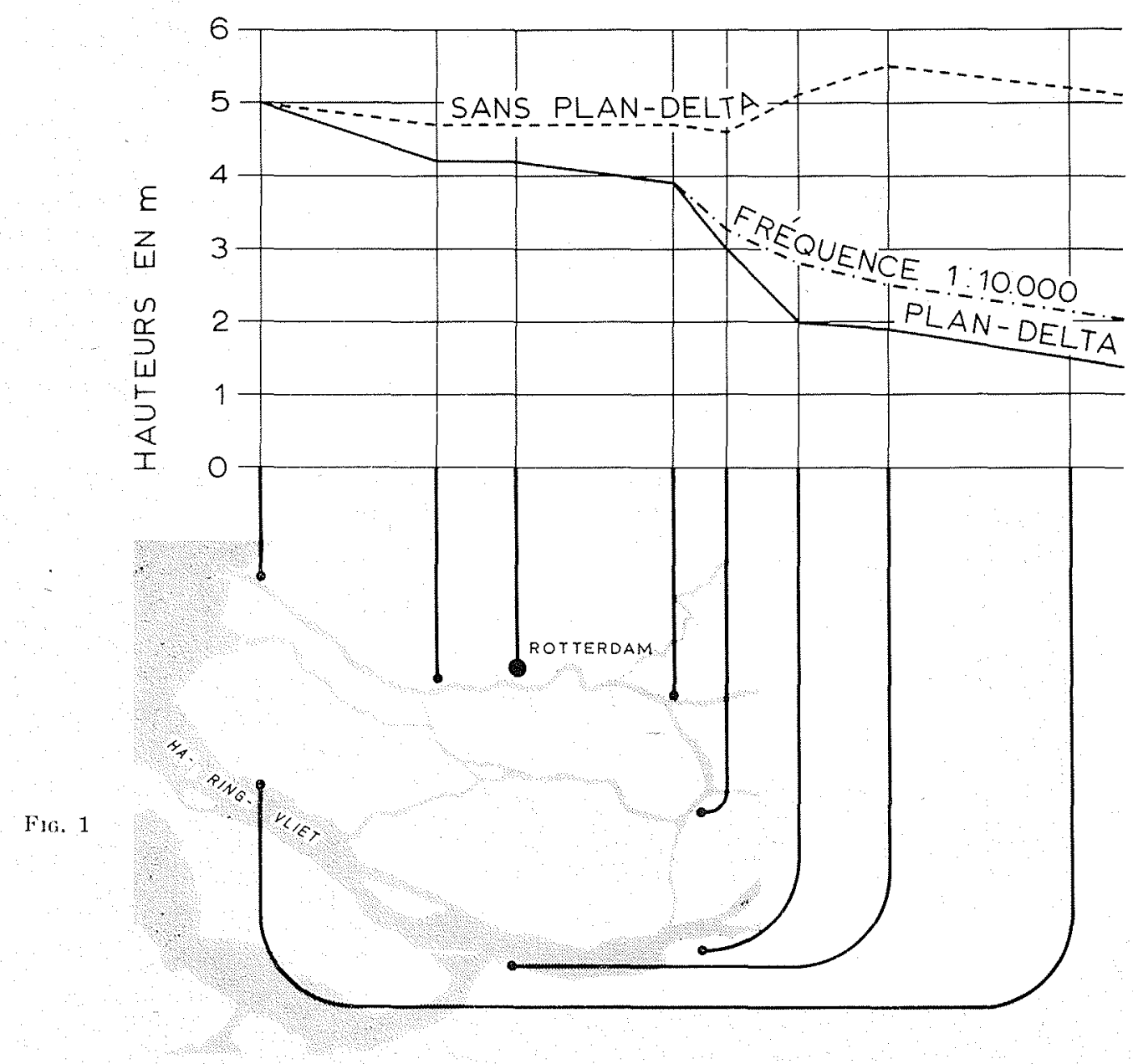

ensemble d'écluses (1) d'évacuation, tandis que les intérêts de la navigation maritime ne permettent pas la fermeture de la Rotterdamse Waterweg.

Il faut prêter attention en premier lieu à la question de savoir où en est l'augmentation de la sécurité contre les inondations dans la région qui garde toujours des contacts limités avec la mer.

L'ensemble des écluses d'évacuation dans le barrage du Haringvliet sera fermé en cas de tempête, de sorte qu'un raz-de-marée ne pourra pénétrer que par la Rotterdamse Waterweg jusqu'au réservoir qui se trouve au nord du barrage du Volkeralk.

Du fait de la capacité absorbante de ce grand réservoir, les raz-de-marée perdront beaucoup de leur force en pénétrant depuis Hoek van Holland dans le pays.

(1) Ecluse = vanne dans certains cas.
La figure 1 donne un aperçu du cours des razde-marée sur les fleuves inférieurs lorsque, avec un débit fluvial qui est normal pendant la saison des tempêtes, un raz-de-marée se produit et atteint le niveau le plus élevé, soit $5 \mathrm{~m}$ au-dessus du niveau moyen de la mer à Hoek van Holland. Les niveaux des raz-de-marée sont portés en ordonnées, tandis que les lieux auxquels se rapportent les niveaux sont portés en abscisse. A l'aide de la partic carte encadrée, on peut suivre la perte d'ampleur que subit l'onde de marée sur son chemin vers le réservoir du Haringvliet. A titre de comparaison, on a aussi indiqué quel serait le cours de ces niveaux si le Projet Delta n'était pas réalisé.

La possibilité d'un raz-de-marée qui atteint $5 \mathrm{~m}$ au-dessus du niveau de la mer se présente une fois tous les 10000 ans (soit $1 \%$ par siècle). Comme, dans les fleuves inférieurs, les débits fluviaux influent également sur les niveaux de haute marée, les combinaisons de raz-de-marée moins élevés et de débits fluviaux plus élevés 
doivent être envisagées. L'influence des razde-marée aura par exemple tellement diminué dans le réservoir du Haringvliet, que, pour déterminer la fréquence des niveaux d'eau élevés, l'influence des débits fluviaux prédominera.

Pour déterminer les cotes de base, on a fixé pour l'ensemble de la région des combinaisons de raz-de-marée et de débits fluviaux telles que la fréquence de la cote de base soit partout la même, soit 1:10000.

Cette figure indique aussi la diminution de ces cotes de base. L'influence qu'exercent les débits fluviaux sur les cotes de base se laisse nettement distinguer dans la région qui est la plus éloignée de la mer. Si l'on compare ces cotes de base avec les niveaux des raz-de-marée tels que ces derniers auraient été sans le Projet Delta, il en résulte que, même en renonçant à lobturation de la Rotterdamse Waterweg, la sécurité est considérablement plus grande. Etant donné que le projet des barrages satisfait notre but principal, d'autres intérêts et d'autres possibilités surgissent pour utiliser dans une mesure aussi intense que possible ces travaux coûteux.

C'est que la fermeture des bras de mer constitue aussi la méthode la plus efficace pour éviter la salinisation croissante dans la région du Delta. Des eaux en plus grande quantité et de meilleure qualité seront disponibles pour l'usage ménager et l'usage industriel, mais surtout aussi pour l'agriculture, où l'on pourra éviter, tout comme les dommages causés par la salinisation, les diminutions de récolte qui sont dues à la sécheresse.

Par suite des fermetures, ledit « Lac Zélandais » se crée au sud du barrage du Volkerak, comme on le voit sur la figure 2. Lorsque

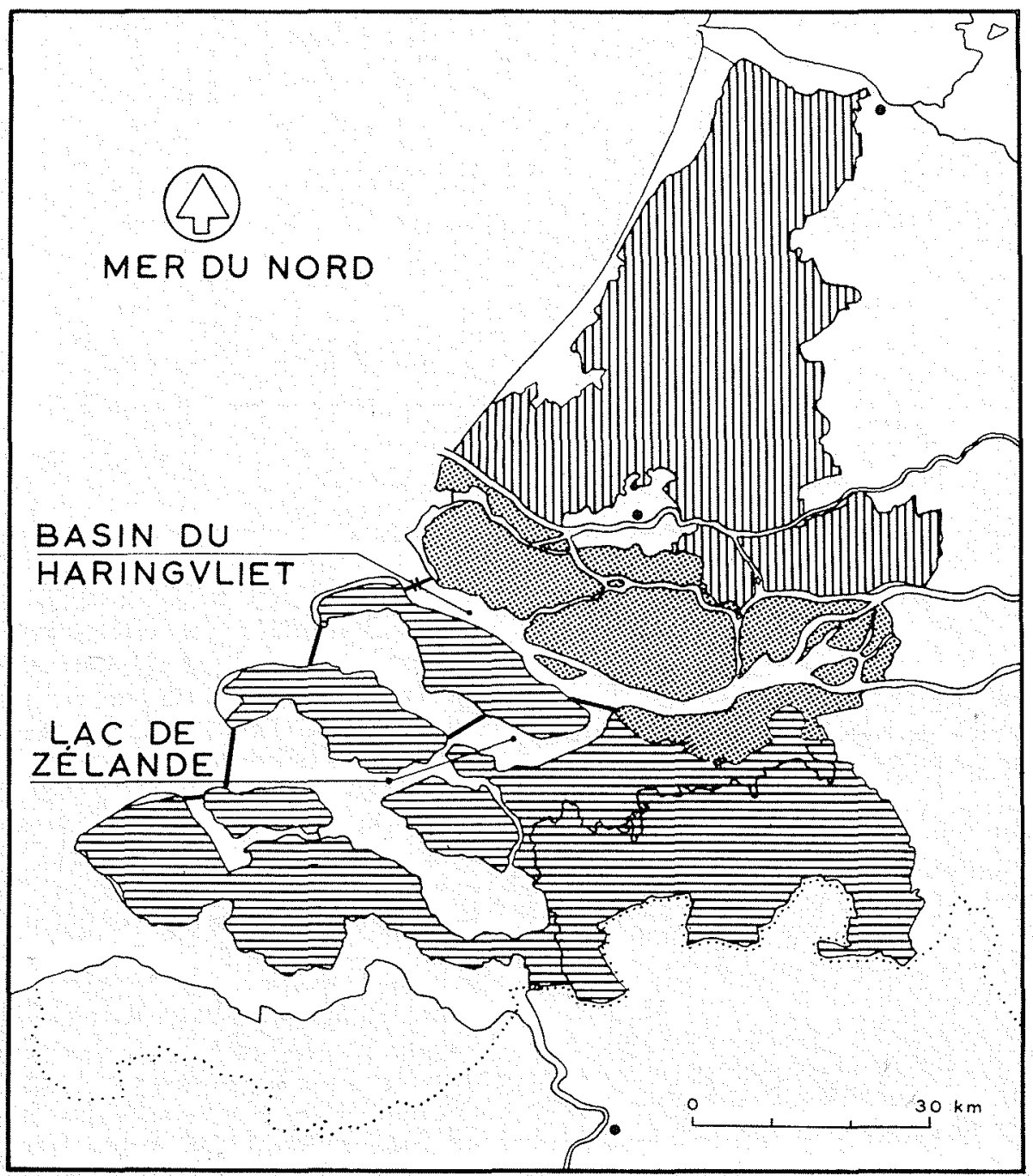

FIG. 2 


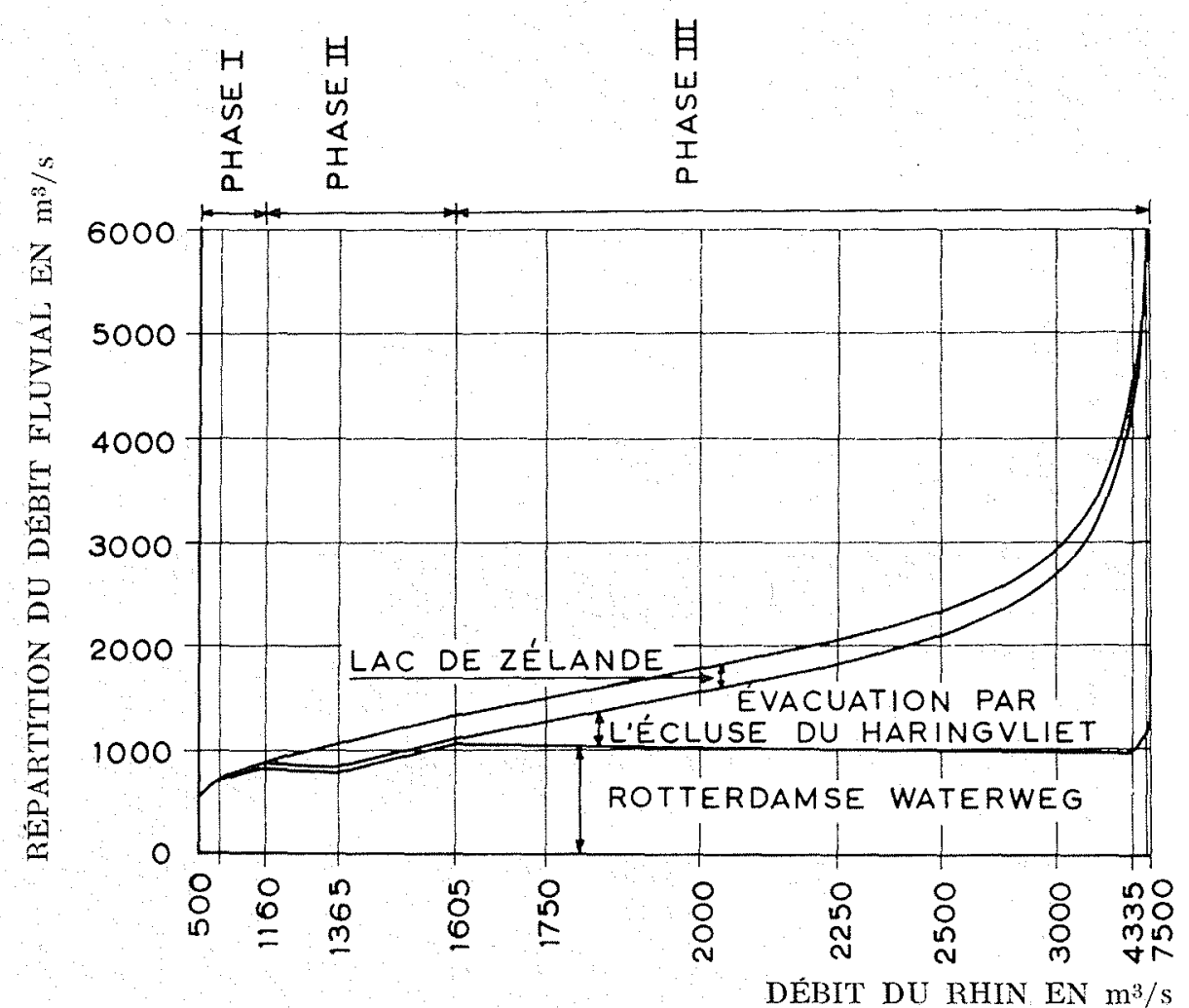

FIG. 3

l'afflux d'eau douce sera suffisant, ce lac se dessalera en quelques années. Il sera alors possible de créer un réservoir d'eau de 500 millions de mètres cubes. Cette réserve suffit pour fournir, pendant près d'un mois environ, $200 \mathrm{~m}^{3} / \mathrm{s}$ d'eau qui serviront, pour la majeure partie, à éviter les diminutions de récolte dans la région d'environ 170000 ha qui est marquée par hachures horizontales sur la figure.

La région au nord du barrage du Volkerak se trouve, comme nous l'avons vu, encore ouverte à la mer, à Hoek van Holland. Il n'est donc pas possible de créer une grande réserve d'eau dans cette région. L'eau du Haringvliet deviendra toutefois entièrement douce et le réservoir servira de pare-choc pour les variations entre l'afflux et l'évacuation de l'eau fluviale. Il sera ainsi possible de pourvoir d'eau depuis le réservoir du Haringvliet, la région d'environ 80000 ha qui est marquée en gris sur la figure.

On peut de plus guider en majeure partie la répartition des débits fluviaux dans le réservoir du nord, parce qu'on peut la régler à volonté à l'aide des écluses du Haringvliet. C'est très important pour la Rotterdamse Waterweg qui est exposée dans une forte mesure à la salinisa- tion et qui a une vaste région à approvisionner, à savoir la région 150000 ha qui est marquée par hachures verticales sur la figure.

En premier lieu, on visera, lors de la répartition des débits fluviaux, à refouler l'eau de mer salée qui pénètre par la Rotterdamse Waterweg. Lorsque les débits fluviaux sont modestes, comme c'est indiqué à gauche sur la figure 3 , le plus d'eau possible sera donc dirigé vers la mer par la Rotterdamse Waterweg.

Une très petite quantité d'eau seulement sera utilisée pour neutraliser l'intrusion d'eau salée, près des écluses du Haringvliet et par quelques autres écluses à sas. Cet état de choses est indiqué sur la figure comme la phase $I$.

Si la quantité d'eau qui est amenée par les fleuves est plus grande que celle qui est nécessaire pour combattre le sel de la Rotterdamse Waterweg, l'eau superflue sert à nourrir et à « laver» le lac zélandais; c'est la phase II sur la figure. Lorsque les débits fluviaux augmentent encore davantage, la phase III montre comment, dans la partie droite de la figure, l'eau superflue sera évacuée lors du reflux par les écluses ouvertes dans le Haringvliet. L'ouverture des écluses est, dans ce cas-là, adaptẻe au 


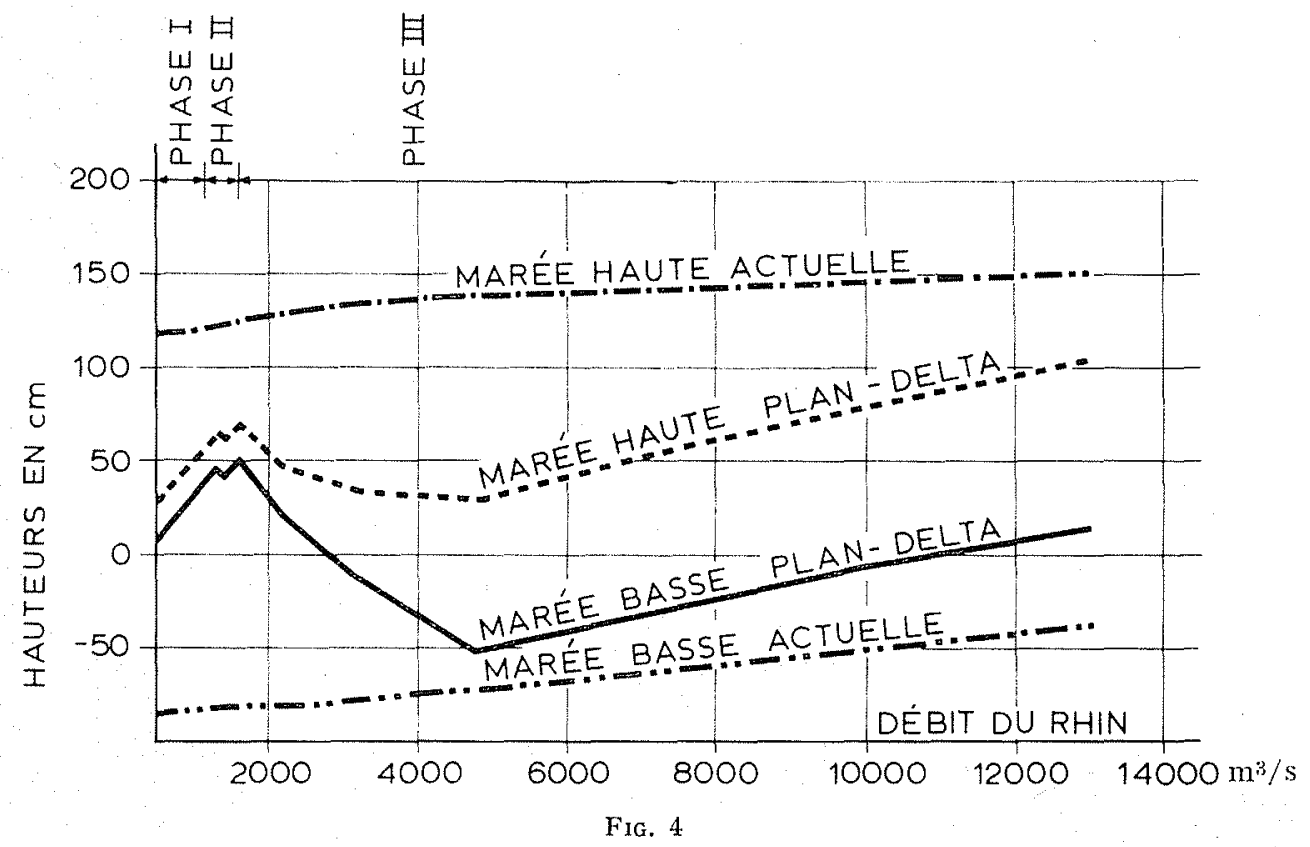

Marées normales dans le bassin du Haringvliet (Willemstad).

débit à évacuer. Ce n'est que lorsque les débits fluviaux seront très importants que les écluses seront entièrement ouvertes lors du reflux, afin d'éviter qu'une trop grande quantité de ces débits ne coule vers la Rotterdamse Waterweg par le Noord. Les vitesses de courant dans le Noord, lors du reflux, pourraient dans ce cas-là entraver considérablement la navigation et attaquer les rives dans une mesure dangereuse.

Il est évident que l'action régulatrice des écluses dans le Haringvliet influera, dans une vaste région du Delta, tant sur les niveaux d'eaux que sur les courants. En ce qui concerne les niveaux d'eau, l'influence des débits fluviaux dans la région du Delta sur ces niveaux est actuellement encore régie par l'influence de la marée. C'est ainsi que, dans la partie est du Haringvliet, par exemple, à savoir, à Willemstad, la marée haute moyenne se trouve à $1,30 \mathrm{~m}$ au-dessus du niveau moyen de la mer, et la marée basse moyenne à $0,8 \mathrm{~m}$ au-dessous du niveau moyen de la mer.

Cet état de choses changera considérablement lorsque les écluses dans le Haringvliet commenceront à fonctionner. En cas de débits fluviaux modestes et lorsque les écluses en question seront entièrement fermées (phase I et phase II), le mouvement des marées ne pourra pénétrer dans la région que par la Rotterdamse Waterweg. Dans ces conditions, les niveaux d'eau de la Rotterdamse Waterweg ne subiront eux- mêmes que peu de changements mais, plus en amont dans la direction du Haringvliet, le mouvement des marées sera graduellement tellement freiné que la différence entre les marées dans le Haringvliet ne sera que de quelques décimètres. En cas de débits fluviaux plus importants, lorsque les eaux superflues seront évacuées lors du reflux par les écluses dans le Haringvliet (phase III), le mouvement des marées dans le réservoir sera renforcé par cette évacuation intermittente. La différence entre les marées augmentera à mesure que les écluses dans le Haringvliet seront, en fonction des débits croissants, davantage ouvertes. Enfin, en cas de débits importants et encore plus considérables, la différence entre les marées sera, à l'intérieur des écluses, de 8 à $9 \mathrm{dm}$. C'est ce que montre la figure 4, où l'on voit que la différence entre les marées est actuellement de l'ordre de $2 \mathrm{~m}$ (courbe supérieure et courbe inférieure), quel que soit le débit du Rhin; tandis qu'après la fermeture $d u$ Haringvliet (courbes médianes), elle sera de 8 à $9 \mathrm{dm}$, soit la moitié de la différence actuelle quand le Rhin débitera $10000 \mathrm{~m}^{3} / \mathrm{s}$ comme cela est représenté à droite, et seulement de quelques décimètres quand le Rhin débitera $1000 \mathrm{~m}^{3} / \mathrm{s}$, comme cela est représenté à gauche.

Les modifications des niveaux de haute-marée et de basse-marée ont des conséquences profondes, de nature variée, qui rendent une adaptation nécessaire. 
FIG. 5

Courants de marée maximum en cas de débit normal du Rhin, avant la réalisation du Plan Delta.

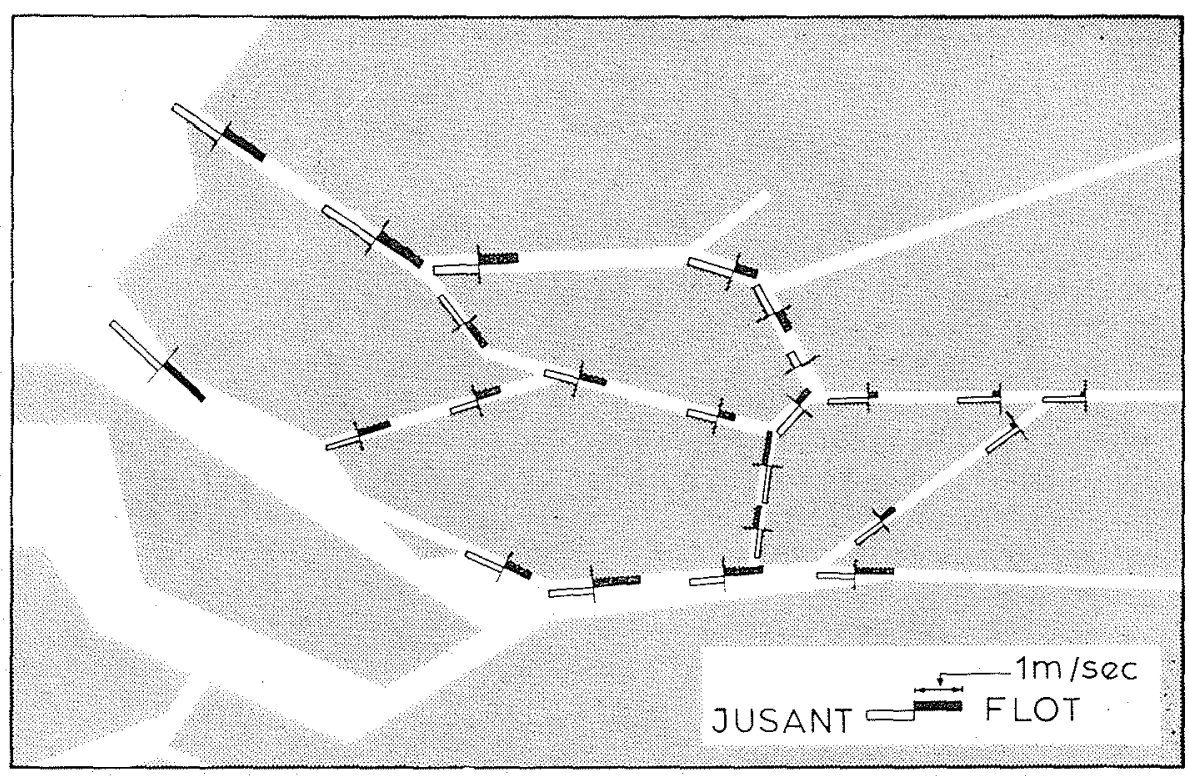

C'est ainsi que, d'une part, des difficultés se présentent par exemple, par l'augmentation des niveaux de basse-marée dans les polders qui, jusqu'ici évacuaient leur débit d'eau lors du reflux entièrement ou partiellement par la voie naturelle et par la force de gravité. D'autre part, l'abaissement des niveaux de haute-marée entraine de nombreux problèmes. C'est que nombre de polders laissent en ce moment entrer librement l'eau douce pour «laver» leurs bassins-réservoirs et pour en régler le niveau d'eau. Il faudra procéder ici aussi à des adaptations. Les petits ports et les écluses dans lesquels on peut entrer actuellement à marée haute devront être approfondis avant que les écluses dans le Haringvliet ne commencent à fonctionner. Les chantiers de constructions navales le long des fleuves inférieurs sont, dans la plupart des cas, adaptés aux niveaux de haute-marée qui se présentent actuellement; leurs cales devront toutefois être allongées de façon à ce qu'elles soient de nouveau en accord avec les niveaux d'eau futurs.

Il est évident qu'avec le changement des niveaux d'eau, les courants aussi se modifieront considérablement. Cela implique que des adaptations doivent également être faites dans les fleuves, à de nombreux endroits. La figure 6 indi-

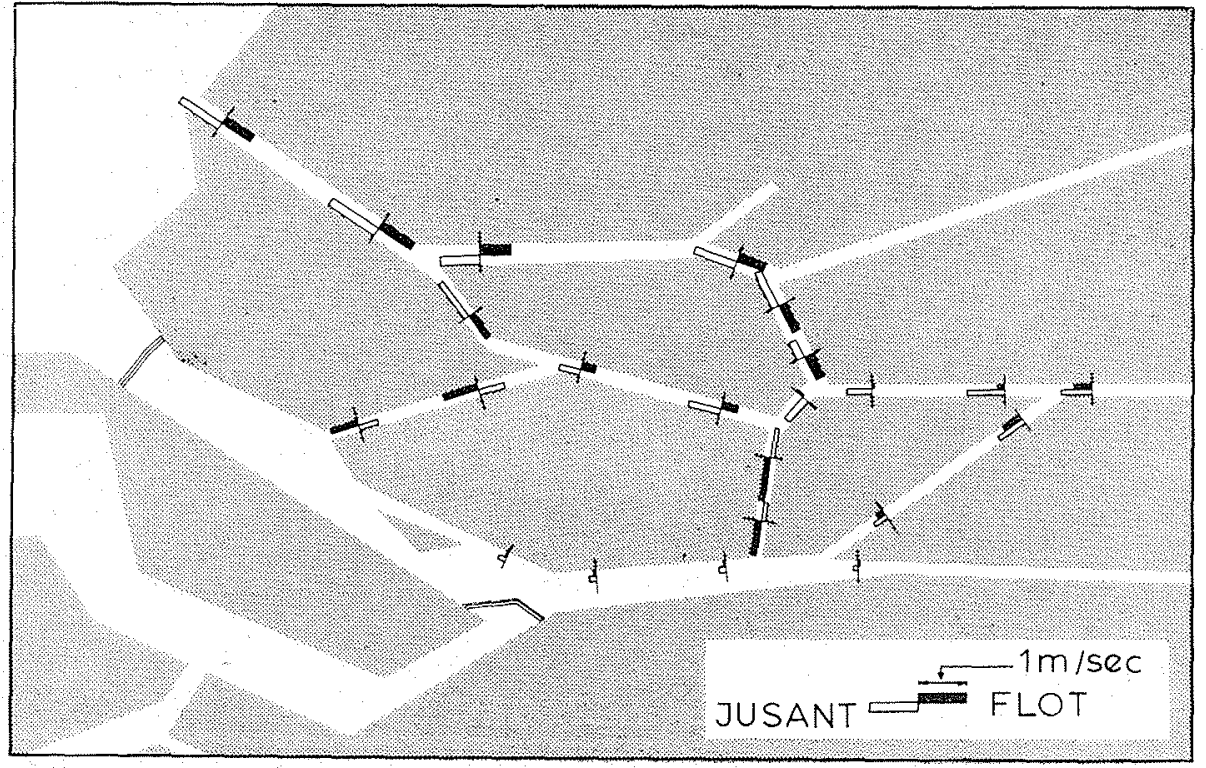

Fig. 6

Courants de marée maximum en cas de débit normal du Rhin, après la réalisation du Plan Delta, 
que, dans le cas d'un débit fluvial moyen, les vitesses maximales du flux et du reflux, telles qu'elles seront après la réalisation $d u$ Projet Delta et en comparaison avec la situation actuelle (fig. 5). Ces figures font, par exemple, remarquer que, dans le Haringvliet et dans quelques fleuves correspondants, le courant de marée montante qui se dirige vers l'intérieur du pays, fait totalement défaut. Cela provient du fait que le mouvement des marées y est pratiquement nul. Ces fleuves prendront ainsi le caractère de fleuves situés dans un bassin supérieur, tandis que les vitesses de courant diminueront sensiblement. Cet état de choses peut entraîner des problèmes pour l'évacuation des bancs de glace pendant les hivers rigoureux.

Il peut se révéler nécessaire dans ce cas-là d'ouvrir entièrement les écluses dans le Haringvliet. tant lors du reflux que lors du flux, et de rétablir ainsi les conditions originales. Or, l'eau salée est alors de nouveau admise dans le réservoir du Haringvliet. Les modifications les plus profondes se produiront toutefois dans les fleuves qui relient latéralement le Haringvliet avec la Rotterdamse Waterweg. C'est que ces fleuves doivent faire passer, comme nous l'avons constaté, beaucoup d'eau dans la direction de la Rotterdamse Waterweg, lors de débits fluviaux importants et même modestes, pour combattre l'eau salée de la mer. qui y pénètre. Le Noord notamment crée de nombreux problèmes parce qu'il forme le chaînon principal du système entier.

La figure 7 indique, aussi bien pour la situation actuelle que pour la situation avec le Projet Delta, les vitesses maximales du flux et du reflux dans le Noord en tant qu'élément du débit fluvial du Rhin supérieur. On verra aussi deux faisceaux de courbes, dans les parties de cette figure relatives tant au flux qu'au reflux, faisceaux qui représentent la relation de ces vitesses avec l'ouverture des écluses dans le Haringvliet et l'écoulement fluvial le long du Noord qui s'ensuit. On constatera que, dans la phase II du programme du barrage, ce sont surtout les vitesses du courant de reflux qui augmentent en forte mesure. La période du courant de reflux augmente par rapport à la période du flux. Ces changements se produisent lors des débits du Rhin habituels. Tout cela rend probable que le sol du Noord sera creusé par l'érosion. En élargissant ce fleuve, on ne supprimera guère cette difficulté, parce que cela aura comme conséquence que le mouvement des marées pourra plus facilement pénétrer jusqu'au réservoir du Haringvliet, tandis que les niveaux moyens baisseront. La réduction

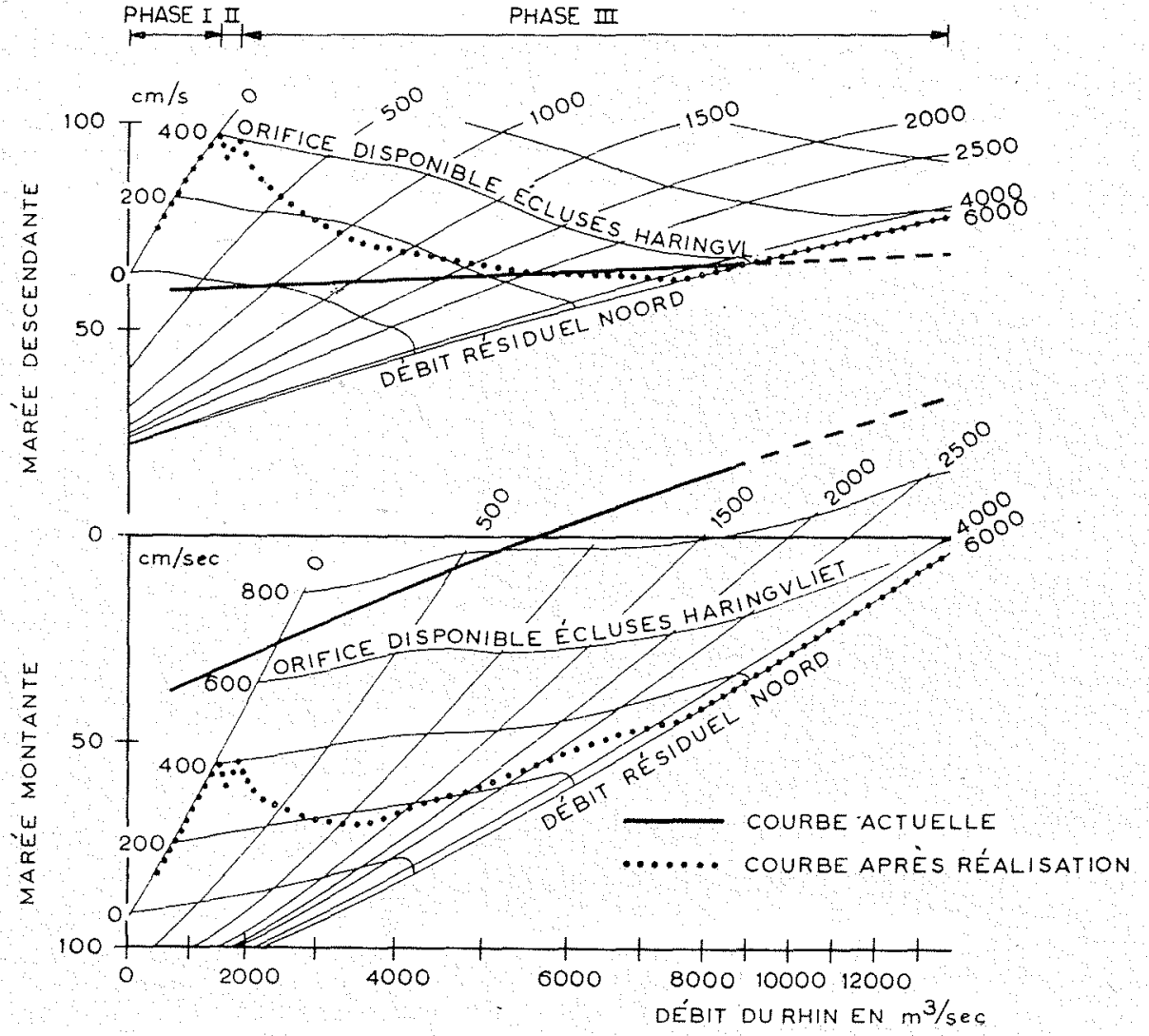

Fic. 7

Courants de marée maximum dans le Noord. 


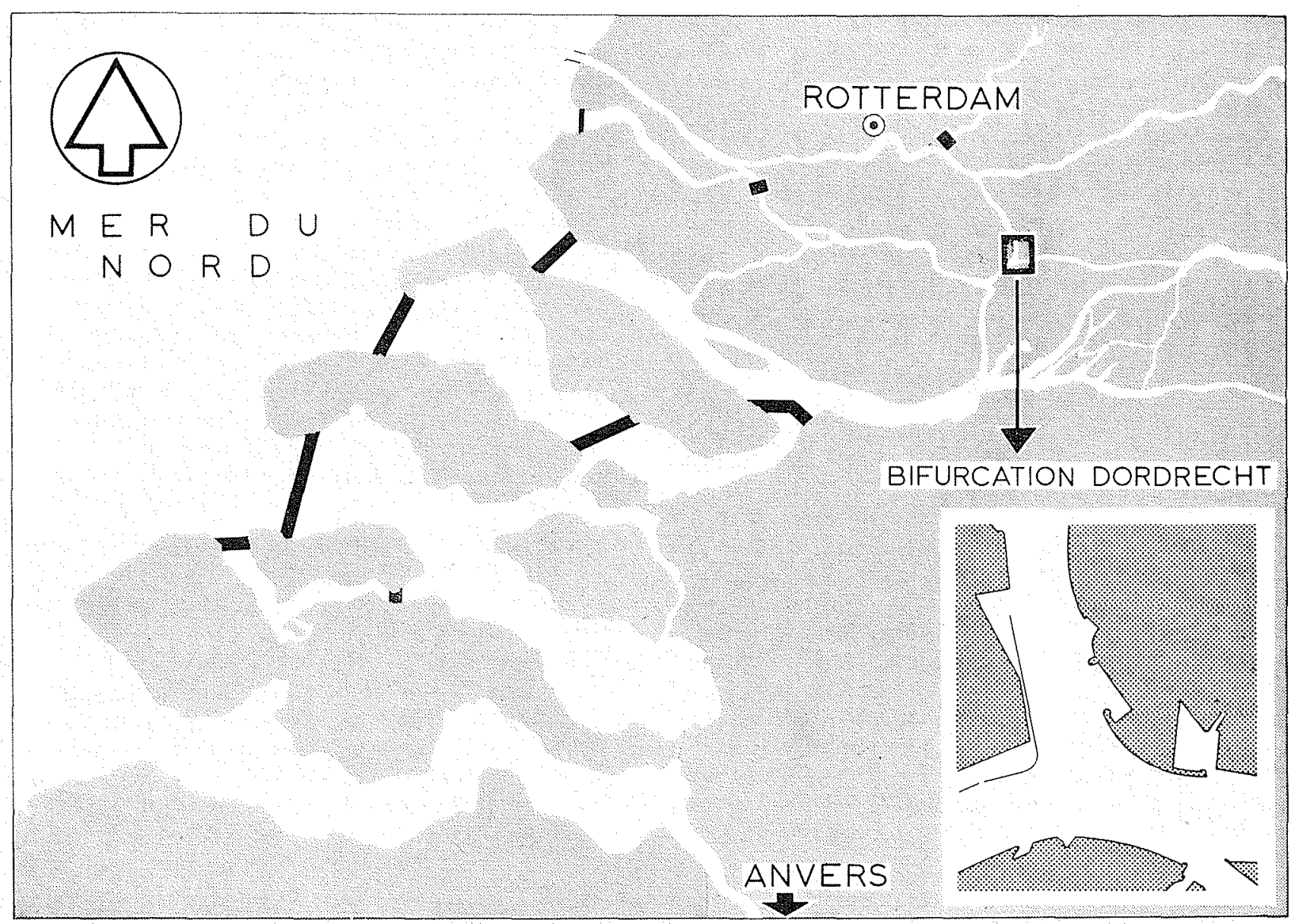

FIG. 8 et 9

des vitesses du courant qui est l'effet d'un profil plus large du fleuve, en sera supprimée pour la majeure partie. Il sera donc probablement nécessaire de protéger le lit de ce fleuve contre un creusement en le couvrant de gravier.

Pour la navigation également, les changements de la situation des courants auront des conséquences. Du fait qu'en plus des vitesses maximales, les différences de phase du courant dans les différents affluents du fleuve changeront, des difficultés pour la navigation sont notamment à prévoir aux points de séparation des différents fleuves.

Je veux attirer maintenant l'attention particulièrement sur un de ces points de séparation, à savoir celui qui se trouve près de la ville de Dordrecht (fig. 8-9) .Ici se rencontrent le Merwede inférieur, un affluent de l'ensemble du Rhin inférieur, le long duquel une large part des débits fluviaux est dirigée, le Noord qui forme un chaînon de l'écoulement des débits fluviaux vers la Rotterdamse Waterweg et la Vieille Meuse qui constitue un chaînon de liaison avec le Haringvliet.
Ce point de séparation se trouve sur la voie de navigation tant de Rotterdam que d'Anvers vers le Rhin, qui appartient à l'une des voies de navigation les plus fréquentées du monde. Plus de 310000 navires passent ici chaque année; ils ont une capacité de charge totale de 125 millions de tonnes. Cela signifie donc une fréquence d'environ 1000 navires par jour ouvrable.

Les constructions denses de la ville de Dordrecht sur l'une des rives et les industries importantes sur les deux autres rendent extrêmement difficile de procéder ici à une amélioration des fleuves qui soit entreprise sur un plan étendu.

On a pu conclure d'une enquête provisoire menée à cet effet, que la situation et, en particulier, les différences de phase entre les courants du flux et du reflux dans les différents affluents, changeraient dans une forte mesure, lors du fonctionnement des écluses dans le Haringvliet. On pouvait prévoir que ces modifications impliqueraient de graves conséquences pour la navigation. 
La figure 10 donne une idée du cours de la répartition des débits entre les différents affluents fluviaux pendant une marée. Sur cette figure, le débit du Merwede inférieur est porté en abscisses et celui du Noord en ordonnées. En raison de la continuité, le débit de la Vieille Meuse est done porté sur un axe à $45^{\circ}$. La direction du courant est donc indiquée grosso modo pour chaque secteur de ce diagramme.

La courbe pleine de cette figure indique le cours de la répartition du débit dans les circonstances actuelles; il n'y a pas de différences marquées quand il y a des variations dans les débits provenant du bief amont. La courbe à traits pleins indique les circonstances après la réalisation du Projet Delta, dans le cas des débits moyens, et le pointillé, dans le cas des débits assez élevés. On remarque que ce sont surtout les débits dans le quadrant en bas à droite, qui augmentent dans une forte mesure en cas d'apports élevés. Dans ce cas-ci, les vitesses de courant sont orientées vers le point de séparation, tant des courants en provenance du Noord que de ceux en provenance du Merwede inférieur. Dans les circonstances actuelles, ces vitesses sont petites, soit de 10 à $20 \mathrm{~cm}$ par seconde. Mais, à l'avenir, elles pourront s'élever à $1 \mathrm{~m} / \mathrm{s}$ et elles seront en même temps, pendant environ $3 \mathrm{~h}$ par marée, plus élevées que $50 \mathrm{~cm} / \mathrm{s}$. Cet état de cho- ses pourra présenter des difficultés pour la navigation. Comme cela a été tracé en grandes lignes sur la figure 11, un navire qui va remonter le Merwede, s'approche du point de séparation, avance d'abord avec le courant mais, arrivé au point de séparation, l'avant du navire se trouve assailli par un courant en sens contraire qui vient de biais. Cette image du courant présentera de grandes difficultés, en particulier pour les convois poussés qui ont une longueur de 175 mètres et qui passent par ce coude déjà accentué. En vue d'étudier ce problème, on a construit un modèle réduit au laboratoire hydraulique « de Voorst», une annexe en plein air du laboratoire de Delft, où sont examinés tant l'écoulement que les mouvements de la navigation. La figure 12 montre une photo de ce modèle réduit.

Les écoulements lors des différentes phases de la marée et les différents débits fluviaux, dans la mesure où ils étaient importants pour cette étude, ont été imposés au modèle comme écoulements permanents.

Dans les expérimentations pour la navigation on doit, pour des raisons techniques, donner la préférence à un modèle non distordu. L'influence du vent sur les navires miniatures dans le modèle en plein air et les conditions de constructions mettent toutefois une limite pratique au

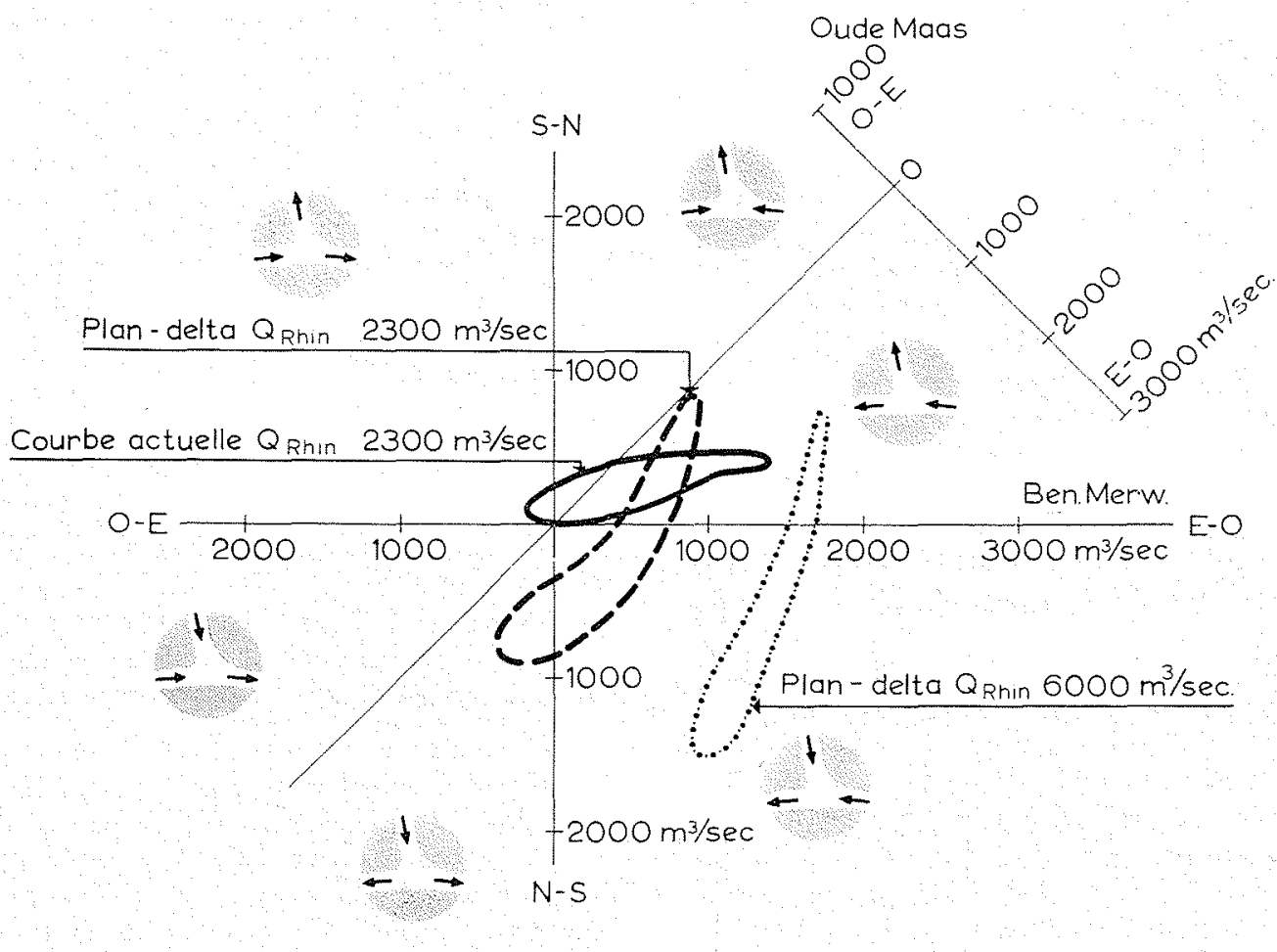

FIG. 10 


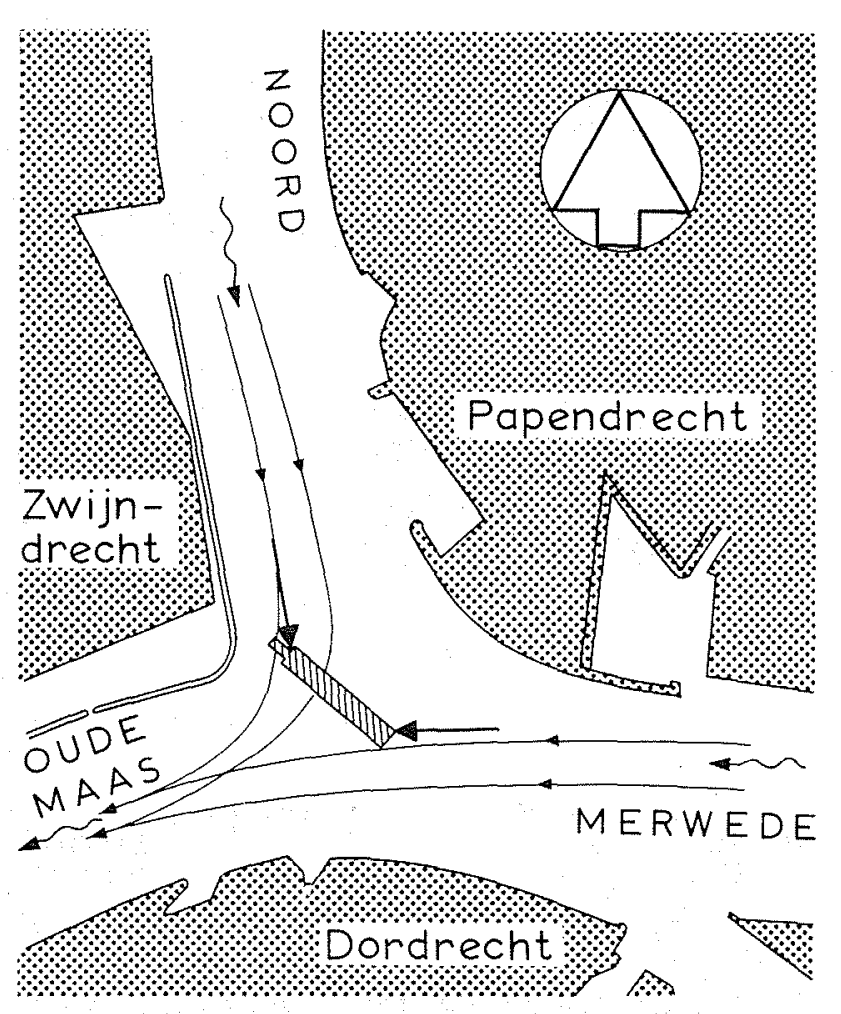

Fic. 11

tirant d'eau minimal des maquettes de navires. Cela entrainerait des dimensions excessives du modèle. Une étude préliminaire avec des maquettes de navire distordues a démontré qu'une légère distorsion était encore acceptable pour l'étude de la navigation. Cela a abouti au choix d'une échelle horizontale de 1:60 et d'une échelle verticale de $1: 40$. Le modèle réduit occupe ainsi une superficie de $100 \times 100 \mathrm{~m}^{2}$. Pour étalonner le modèle en vue de déterminer la répartition exacte de la rugosité sur les parois et sur le sol, on a mesuré le courant, en vitesse et en direction, à vingt-quatre endroits simultanément, pendant un cycle de marée de $13 \mathrm{~h}$. Pour examiner les mouvements de la navigation, on a construit des maquettes du pousseur «Olivier van Noort» avec quatre barges, ainsi que du navire remorqueur à moteur «Aquitania» avec deux chalands, et on les voit sur la figure 13 . Un remorqueur a également été construit avec un certain nombre de chalands remorqués. Des essais ont toutefois montré que le convoi poussé traversait le moment le plus critique en naviguant sur le point de séparation, de sorte que, pour les examens ultérieurs, on a fait principalement des essais avec le pousseur.

Les hélices et les gouvernails étaient commandés par des électromoteurs qui étaient alimentés depuis la rive. On réglait la direction à prendre en dosant le courant électrique. Le câble électrique à branches multiples qui était nécessaire à cet essai était conduit vers le navire à l'aide d'une canne, de telle facon que le navire n'eût à subir l'influence d'aucune force extérieure. Les possibilités d'examiner la navigation sont limitées par l'effet produit par l'échelle. Celui qui commande les moteurs de la maquette peut, par exemple, augmenter plus vite le nom-
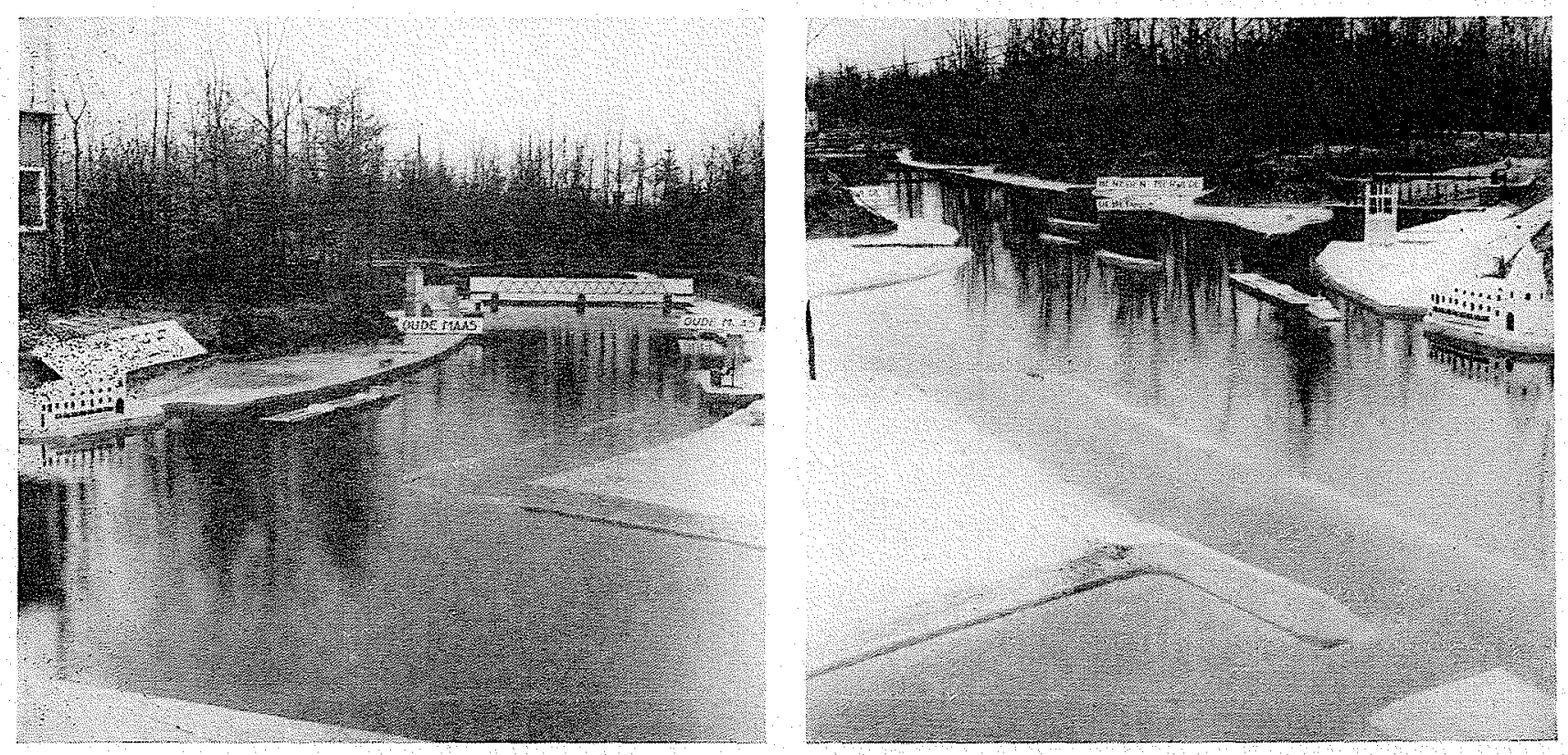

FIG. 12

La navigation à Dordrecht en modèle réduit. 
bre des tours que son collègue ne peut le faire dans la réalité; l'accélération n'est donc pas réduite à l'échelle. La diminution de la vitesse présente la mème difficulté par l'influence qu'exerce la tension de surface dans la lame soulevée par l'étrave. Pour ces raisons, on a réduit au mininum l'utilisation de moteurs dans la maquette.

Une autre difficulté pour le «commandant» de la maquette c'est l'échelle du temps, ce qui fait qu'il devrait apercevoir, décider et agir plus vite que son collègue ne devra le faire en réalité. Bien que la conduite d'une maquette de navire soit plus facile que celle qui est possible dans la pratique, comme cela se voit aussi sur la figure 14, cela complique la navigation de la maquette en comparaison de la réalité. L'échelle du temps est donc l'échelle de la longueur divisée par la racine de l'échelle de la profondeur $=(60 / \sqrt{40})=$ environ 10 .

La valeur des essais effectués pour la navigation se trouve toutefois dans le fait de rendre

F1G. 13

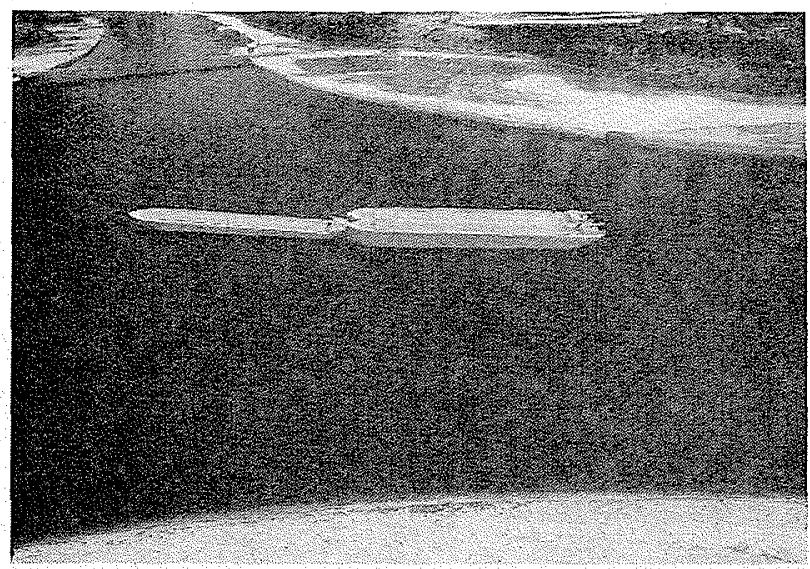

Remorque composéc «Aquitania».

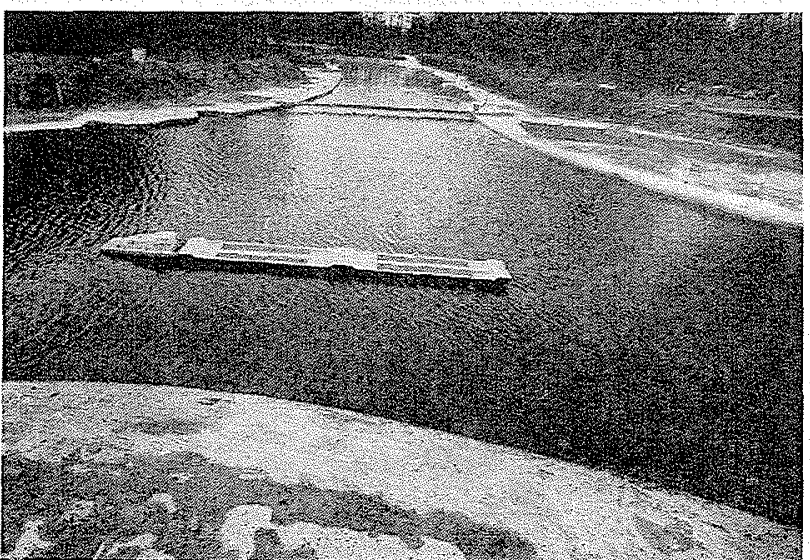

Convoi pousse.

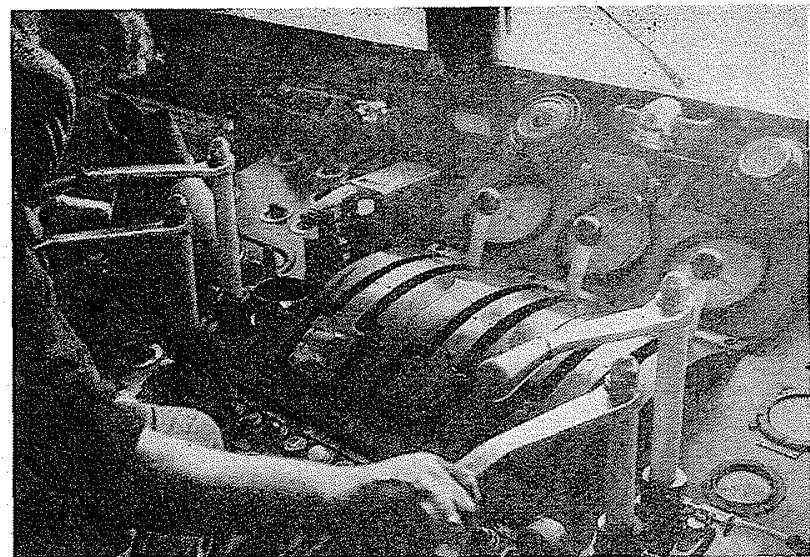

Pilotage d'un convoi poussé.

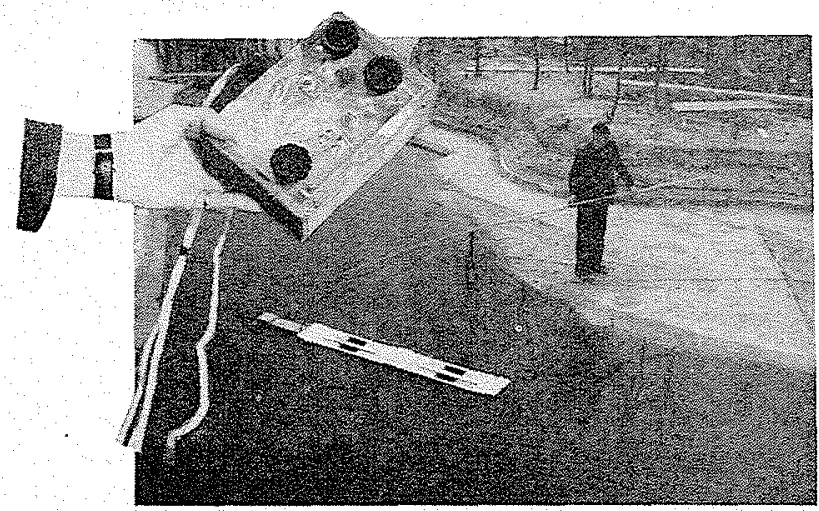

Le même en modèle réduit.

Fig. 14

visible le mouvement, lorsque le «commandant» de la maquette réussit à faire, après de longs exercices et sans se laisser influencer par les échelles de la profondeur, une libre manoeuvre qui pourrait se présenter aussi en réalité. Il faut toutefois, a cet effet, une connaissance parfaite des mouvements de la navigation telle qu'elle est en réalité et une collaboration étroite avec les experts et les bateliers.

On a fait pour cela un grand nombre d'observations pour fixer les mouvements de la navigation à ce point de séparation, lors des différents écoulements. Comme, du fait de l'intensité de la navigation, il n'a pas été possible de déterminer, à l'aide de théodolites, le lieu où se trouvaient les navires, toutes les mancuvres ont été déterminées à l'aide de radar.

En photographiant à de petits intervalles l'image radar d'un ensemble de radar fluvial, on peut reconstruire un image vivante des mouvements de la navigation.

A l'aide des itinéraires qu'on suit dans la réalité, on a réussi à reproduire les manouvres. 

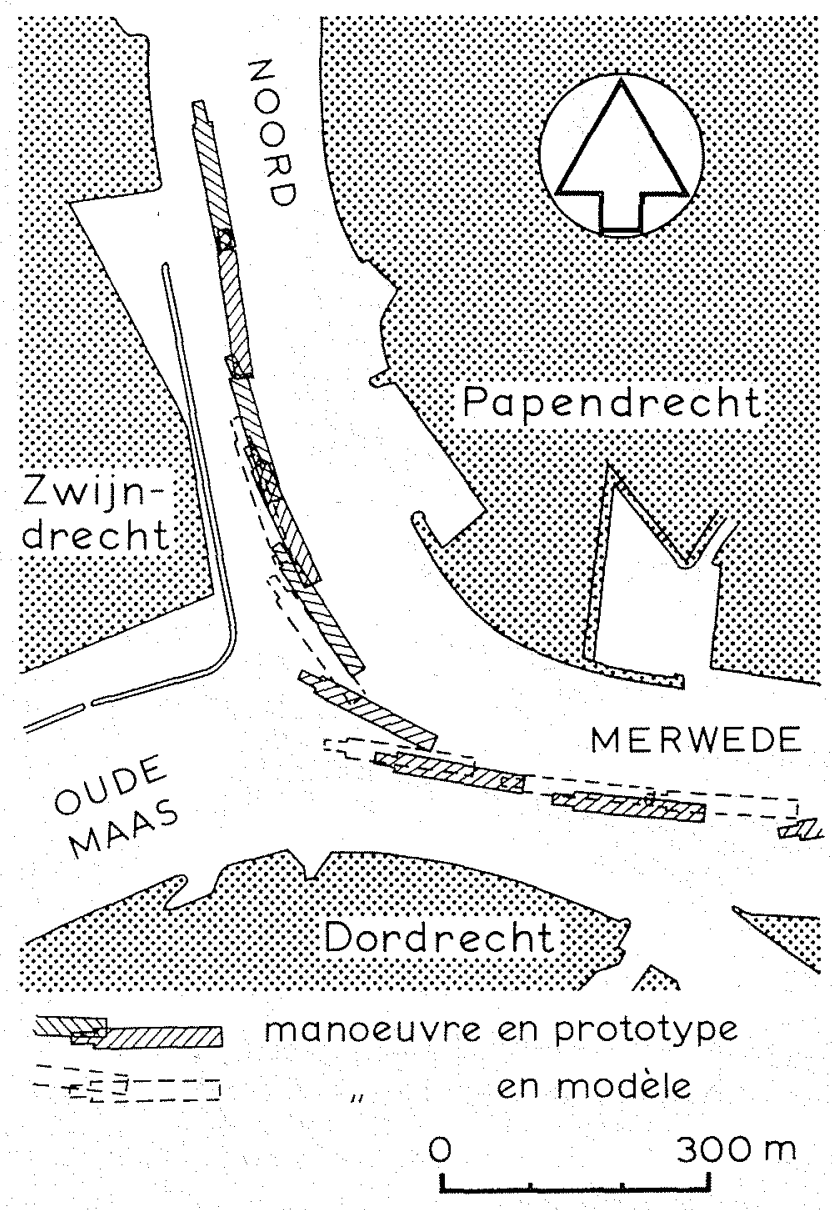

Fig. 15

dans le modèle. Les observations de mouvements dans le modèle ont été faits en photographiant, également à de petits intervalles, la maquette du haut d'une tour de $12 \mathrm{~m}$.

Le « commandant» de la maquette a en outre effectué plusieurs voyages avec un vrai convoi poussé et les navigateurs du pousseur ont essayé leur routine dans le modèle. Après quelque entraînement, le commandant de la maquette était en mesure d'arriver a une navigation qui correspondait fort bien avec la réalité. Sur la figure 15, on voit, l'une dessinée sur l'autre, une route naviguée sur le modèle et une route naviguée en réalité.

L'étude de la situation en partant de la position actuelle des rivages, mais avec les circonstances défavorables des courants dans l'avenir, a appris que les prévisions des difficultés pour la navigation sont en effet justifiées.

La figure 16 montre l'évolution d'une manouvre normale pendant que le navire descend le fleuve, lorsqu'il échoue sur la rive gauche. $\mathrm{La}$ seule méthode pour éviter cette difficulté a été de diriger le convoi pratiquement en travers du courant, avant qu'il ne fût arrivé au point de séparation. Il s'ensuivrait toutefois que toute la voie navigable serait bloquée lors d'tune telle manœuvre, ce qui est évidemment inadmissible.

Le trafic montant au point de séparation, en haut sur la figure, est poussé sur la rive près de Dordrecht, à cause du courant en sens contraire qui assaille l'avant du navire. Une esquisse du courant que l'on voit sur la figure 17 , indique que le courant qui vient du Noord, se détache près de la rive de $Z$ wijndrecht et qu'il passe sous forme d'un tourbillon intense vers la rive sud du fleuve, où des vitesses très élevées du courant d'environ $2 \mathrm{~m} / \mathrm{s}$ se produisent alors.

FIG. 16

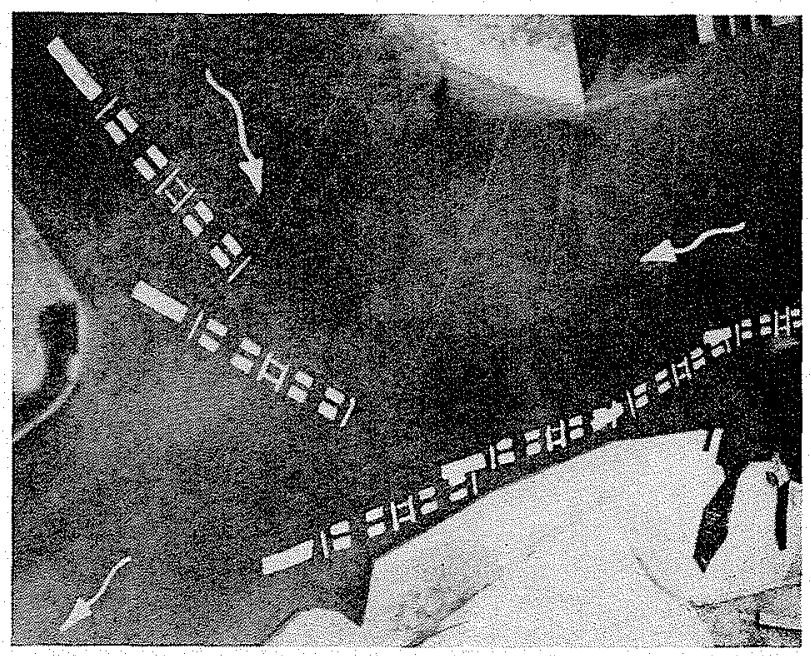

Navigation montante.

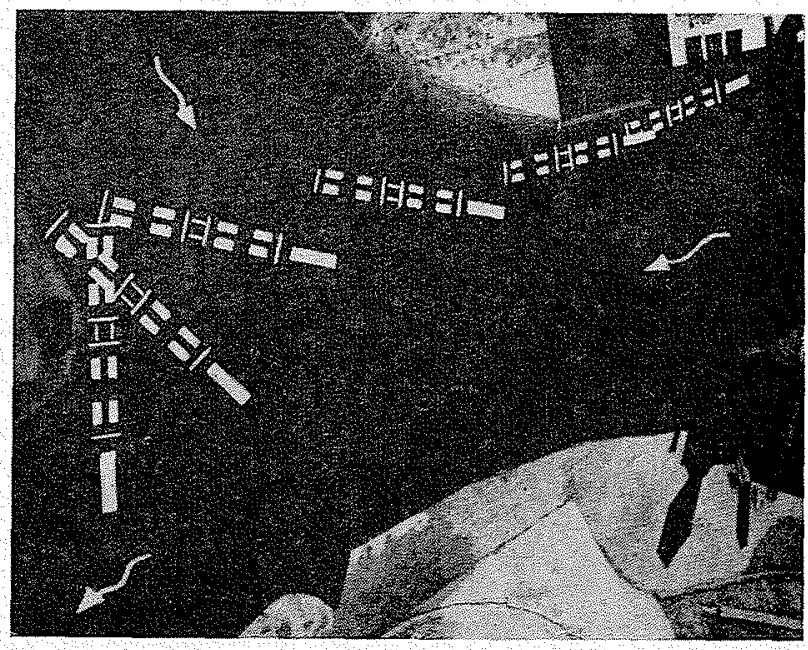

Navigation descendante. 


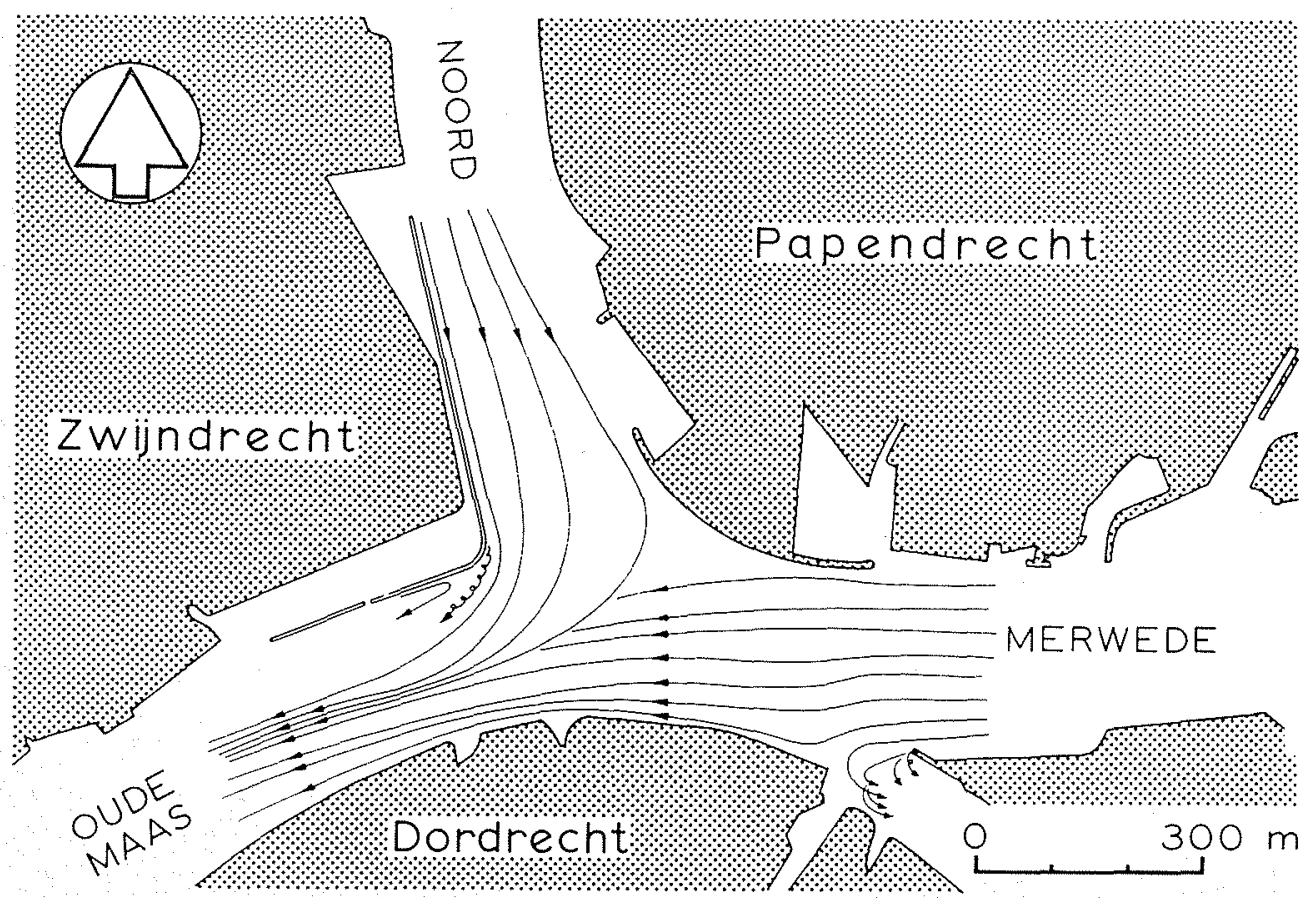

FIG. 17

D'abord, on a cherché à ce propos à améliorer la rive de Zwijndrecht. L'arrondissement jusqu'à un rayon de $700 \mathrm{~m}$ avait comme conséquence que le courant continuait à suivre convenablement cette rive de sorte que des concentrations de courant furent évitées. On le voit sur la figure 18. On pent constater en outre que, de l'au- tre còté de la rive de Papendrecht, une région avec de très petites vitesses de courant s'est créée.

Le trafic montant trouve, plus facilement dans ce cas-ci, assez d'espace pour tourner et peut, à cause de l'écoulement favorable, passer progressivement du courant qui le porte au courant qui

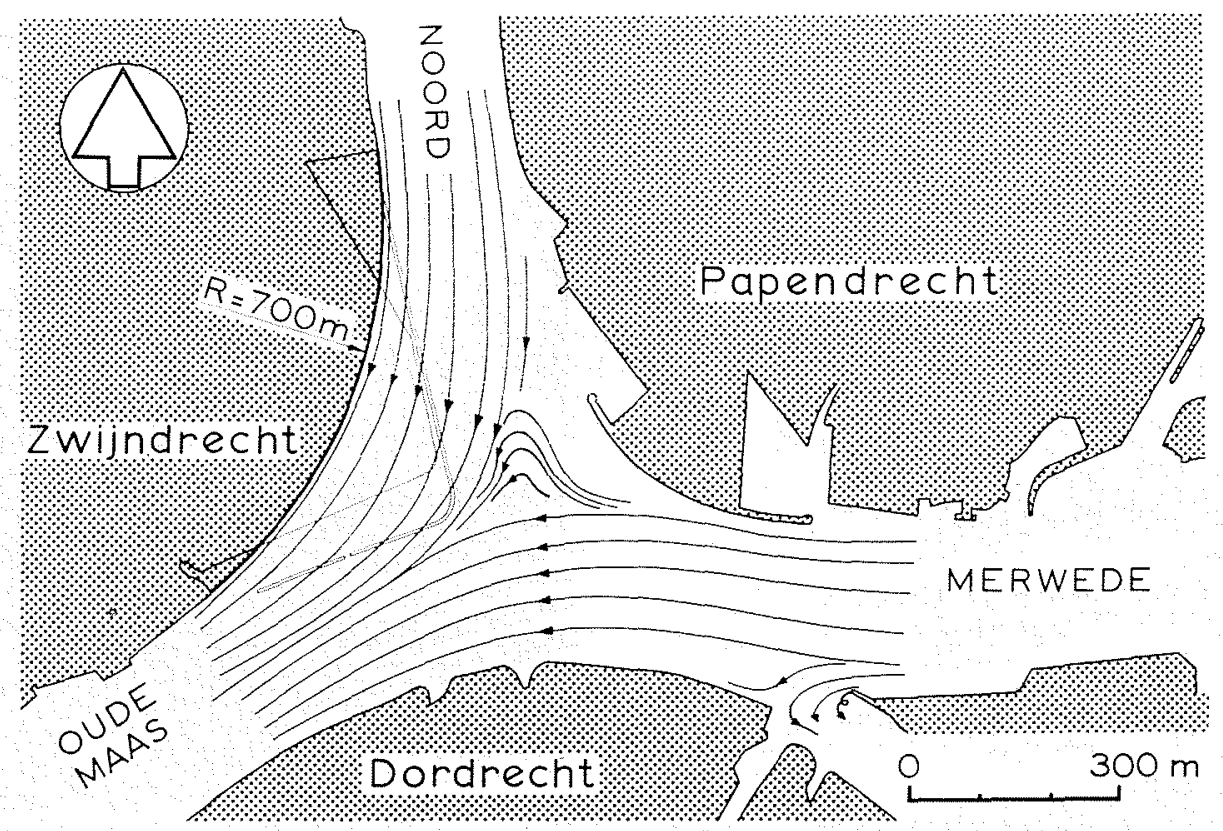

Fig. 18 
arrive en sens contraire. La figure 19 montre une manœuvre du trafic montant qui se déroule bien. Le trafic descendant peut faire usage de la région sans courant que je viens de citer, mais, comme la figure le montre, cette région est encore trop petite et, bien que le danger d'échouer sur la rive soit considéralement réduit, le convoi poussé arrive encore bien près de la rive gauche en dépassant le milieu du fleuve, ce qui risque de produire des collisions avec le trafic qui vient de la direction opposée. Il s'est révélé qu'un arrondissement avec également un rayon de $700 \mathrm{~m}$, à la rive de Papendrecht, comme la figure 20 le montre, avait comme résultat une telle extension de la région sans courant que le trafic descendant pouvait se rétablir ici pour passer du courant dans lequel il naviguait à celui qui vient en sens contraire.

On voit sur la figure 21 que tant le trafic montant que le trafic descendant peuvent sans difficulté passer par ce coude. Pour achever cet examen, la solution a encore été contrôlée dans un grand nombre d'autres configurations du courant qui peuvent encore se présenter à cet endroit, pendant d'autres phases de la marée et avec d'autres débits fluviaux. Aucune difficulté ne s'est toutefois présentée à ce sujet. J'ai consacré une partie importante du temps disponible à un exposé sur ce seul point de séparation. J'espère que j'ai bien expliqué au cours de mon introduction que ce point constitue seulement un exemple caractéristique des nombreuses précautions qui sont à prévoir au cours des travaux dans la région du Delta.

Heureusement, il se révèle que la plupart de ces précautions conduisent en même temps à une amélioration des circonstances existantes.

C'est donc ainsi que le Projet Delta, en plus

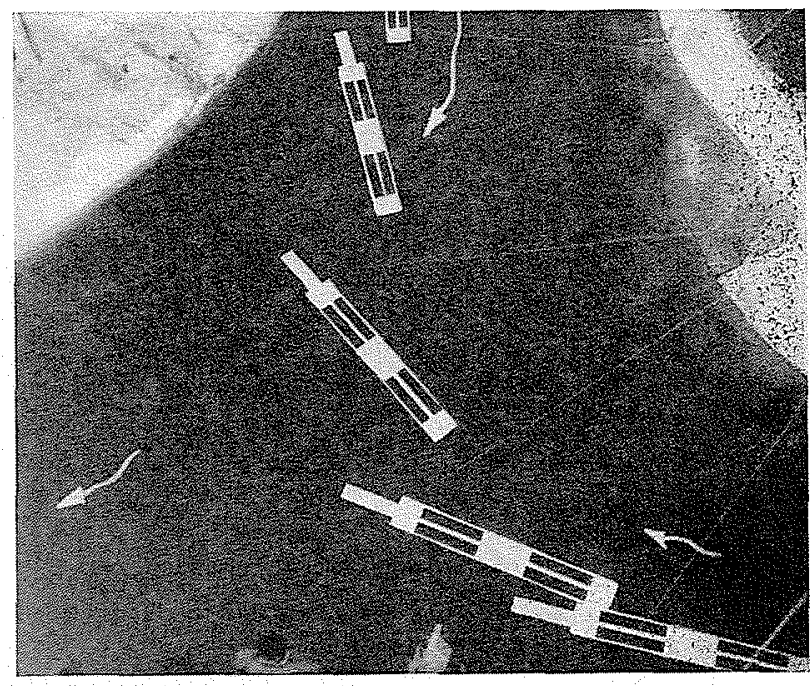

Navigation montante.

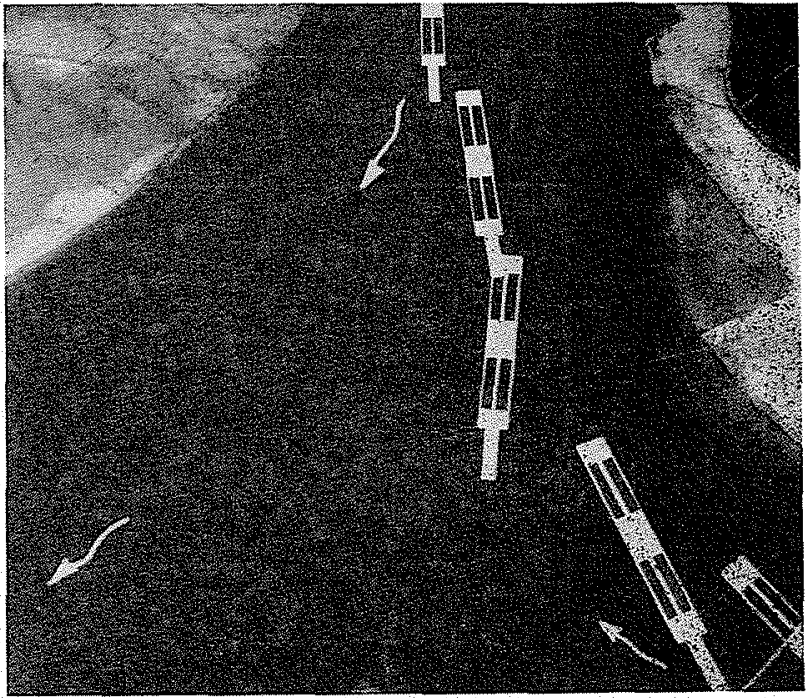

Frg. 19 Navigation descendante.

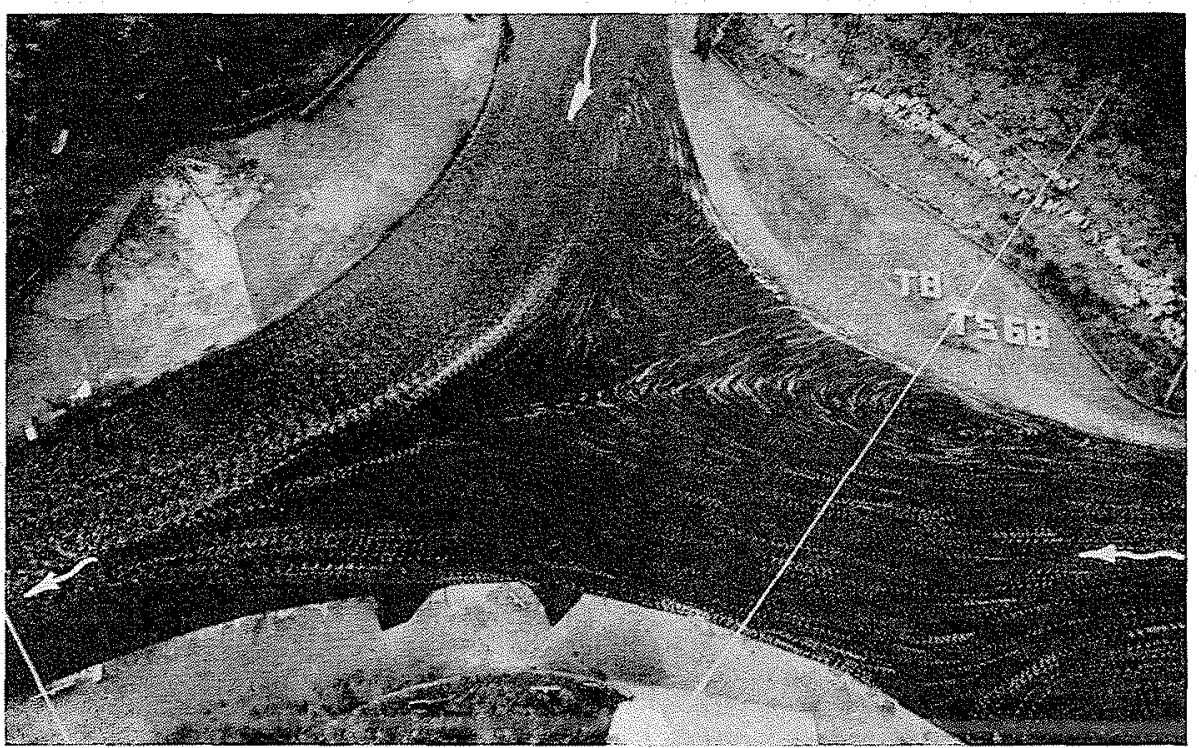



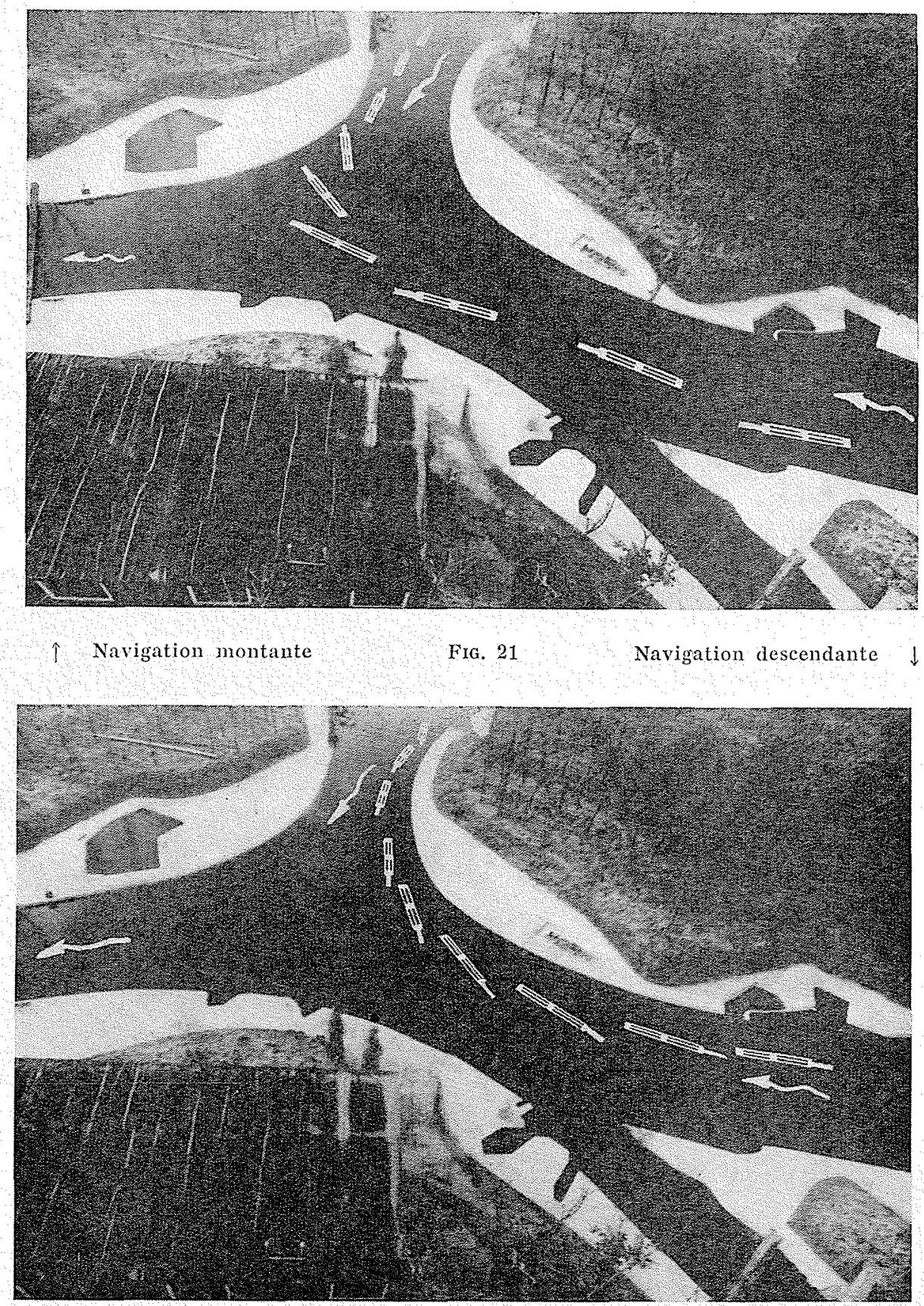

d'une sécurité plus grande contre les raz-demarée, créera, encore de grands avantages relatifs à la distribution des eaux nécessaires à l'agriculture, aux communications terrestres et, par voie d'eau, à la récréation, etc.

Il est donc évident que non seulement la réalisation des travaux du Projet Delta ne changera pas de fond en comble la carte topographique des Pays-Bas, mais aussi que le bienfait de ces travaux s'étendra sur la structure économique du pays entier.
Pour finir, je veux montrer encore un film très court sur les essais de navigation dans le modèle. On verra nettement le fonctionnement des maquettes des navires et les itinéraires qu'ils suivent. On verra d'abord quelques images de la mauvaise disposition des courants le long des rives existantes et, ensuite, le déroulement des choses lors de la solution finale. Je crois que ce petit film sera, en lui-même, assez clair. D'ailleurs, pour changer d'orateur, M. Brandeler reprendra la parole lors de la présentation. 


\title{
DISCUSSION
}

\author{
Président : M. Duffaut
}

M. le Président remercie M. Ounshoons de sa conférence intéressante et parfaitement illustrée.

Sur une question de M. le Président, M. Ounshoon précise que le débit normal moyen du Rhin est de $2200 \mathrm{~m}^{3} / \mathrm{s}$; que le débit minimal est de $700 \mathrm{~m}^{3} / \mathrm{s}$ et le débit maximal de $8000 \mathrm{~m}^{3} / \mathrm{s}$, débits qui ne se produisent qu'un jour par an. Le débit «maximum maximorum» est de $13000 \mathrm{~m}^{3} / \mathrm{s}$.

M. Chapouthrer demande si l'on a étudié les questions des fronts de salinité : comnait-on bien l'influence que va avoir la réalisation du Plan Delta sur la modification de ec front de salinité, de séparation entre les eaux douces et les eaux salées?

M. Oudshoonn répond que cette question a été étudiée et se trouve exposée dans le rapport final du Comité du Plan Delta.

M. VAN DEN BRANDEler dit que, a part la sécurité contre les inondations, la question de la salinité est la question principale pour laquelle on va réaliser le Plan Delta; ceci est surtout vrai pour le bassin septentrional (la réalisation d'un réseau de connections routières entre les îles est un autre facteur de grande importance).

M. Povmanin a noté que le tracé des deux rives avait une courbure de $700 \mathrm{~m}$ de rayon. Est-il prévu un raccordement à l'origine et aux deux raccordements du tracé?

M. Oudshoon dit que le tracé est un cercle sans raccordement.

M. Pechene voudrait savoir pourquoi l'on a choisi les etudes du carrefour de navigation sur un modèle distordu alors qu'un modele non distordu semble a priori plus simple.

M. VAN DEN BRANDELelk dit qu'on doit étudier principalement la profondeur à grande échelle en raison de la rugosité et, dans l'espèce même, en raison surtout du tirant d'eau minimal des maquettes.

M. Grinaver demande pour quelle raison les auteurs du projet Delta n'ont pas envisagé de contrôler les débits des voies d'eau qui font communiquer le Rotterdam Waterweg avec le Haringviet, au moyen de barrages mobiles et d'écluses.

M. VAN DEN BRANDeler dit que la raison en est le prix qui serait alors trop élevé par rapport aux avantages, sans parler du fait que le Noord est un itinéraire de navigation très important que l'on ne peut compromettre par la construction de vannes; il faut que la voie reste libre.

Du reste, grâce à l'ensemble des vannes du Haringvliet et du Volkerak, on dispose d'un dispositif efficace pour contrôler les débits quí s'écoulent vers Rotterdam.
M. BANal souligne la difficulté due au fait que, dans la nature, le pilote est dans la cabine, alors que, sur modele, le pilote a une vue nettement plus haute. Le résultat obtenu, faire suivre, gràce à une expérience, la même route par le modèle et dans la nature, lorsque les conditions hydrauliques sont très différentes, est-il celui que l'on recherche?

M. VAN DEN BraNDELER indique que pour pallier la manière différente de voir les choses, sur modèle réduit et en nature, le timonier du modèle a fait un voyage sur le bateau et le timonier du bateau a fait les essais sur modèle. Tout ce que l'on a trouvé sur modèle sera traduit plus tard, en nature, par balisage.

M. Baral se demande si l'on ne pourrait pas résoudre la difficulté en mettant une caméra de télévision sur le bateau.

M. VAN DEN Brandezer rappelle toute l'importance de ce carrefour de navigation. Toute la batellerie descendant de l'Allemagne arrive à l'est par le Merwede inférieur. On doit choisir, soit de descendre vers la Belgique et la France du nord, soit remonter vers Rotterdam. De plus, il y a des installations portuaires maritimes qui ont une influence sux l'amont de la navigation puisqu'il y a uno voie directe qui relie le port de Dordrecht à la mer du Nord. C'est cette porte de séparation dont on a parlé et qui est représentée sur le film.

La séance continue par la projection du film sur les recherches sur modèles réduits dans le domaine de l'amélioration des fleuves au profit de la navigation. Ce film est commenté par M. VAN DEN BRANDELEn.

Le carrefour de Dordrecht est montré en nature, sur modèle avant les essais et sur modèle après que la situation a été rectifiée.

Après la projection du film, M. Remenienas demande s'il y a un risque de dépôt de matériaux dans la zone d'eau calme du carrefour de Dordrecht aménagé.

M. VAN DEN Branderler dit qu'en réalité, à cet endroit, la rivière a tendance à s'affouiller. Il $n^{\prime} y$ avait pas de courant dans cette zone, car le film a été pris en marée descendante, mais le régime est intermittent ef l'apport de matériaux transportables dans un régime serait défait pendant l'autre régime.

Le calme dans cette zone ne se produit que pendant quinze jours par an et, là encore, pendant trois heures par marée, ce qui fait espérer que les dépôts seront peu importants.

M. le Président remercie les conférenciers hollandais de leurs explications.

Cette discussion est suivie de la projection du film «Terres des Eaux» (voir compte rendu général des séances).

M. le Président traduit la vive appréciation par l'assistance de la valeur artistique et sociale de ce film.

La séance est levée à 12 h 25 . 